\title{
Clinical relevance of virulence genes in Campylobacter jejuni isolates in Bahrain
}

Correspondence

Abiola C. Senok

abiolacs@agu.edu.bh

Received 4 January 2006

Accepted 13 March 2006

\author{
Ali Al-Mahmeed,† Abiola C. Senok,† Abdulrahman Y. Ismaeel, \\ Khalid M. Bindayna, Khaled S. Tabbara and Giuseppe A. Botta
}

Department of Microbiology, Immunology and Infectious Diseases, College of Medicine and Medical Sciences, Arabian Gulf University, PO Box 22979, Manama, Kingdom of Bahrain

\begin{abstract}
There are no data describing the genetic make-up of Campylobacter strains (an important aetiological agent of diarrhoea) circulating in the Arabian Gulf region. Here, the molecular characterization of two virulence genes in Campylobacter jejuni from Bahrain and the relationship with clinical infection are reported. Molecular screening for cytolethal distending toxin ( $c d t B$ ) and invasion-associated marker (iam) genes was carried out on C. jejuni stool isolates collected from January 2002 to January 2004 in Bahrain. The molecular characterization was correlated with the patients' socio-demographic and clinical parameters. Of the $96 \mathrm{C}$. jejuni strains tested, 50 $(52 \%)$ were $c d t B^{+} /$iam $^{+}, 30(31 \%)$ were $c d t B^{+} /$iam $^{-}$and $16(17 \%)$ were $c d t B^{-} /$iam $^{-}$. Sixty-nine per cent (66/96) of patients were less than 3 years old, with significantly higher detection of $\mathrm{cdtB}^{+} / \mathrm{iam}^{+}$and $\mathrm{cdt} \mathrm{B}^{+} / \mathrm{iam}^{-}$strains $(P<0.001$ and $P<0.01$, respectively $)$ in this age group. Seventy patients $(73 \%)$ were symptomatic. In the group that were less than 3 years old, 62 and $85 \%$ of those with $c d t B^{+} /$iam $^{+}$and $c d t B^{+} /$iam $^{-}$strains, respectively, were symptomatic compared with $100 \%$ for those over 3 years of age. However, the presence of $\mathrm{cdtB}^{-} / \mathrm{iam}^{-}$ strains still resulted in clinical infection in the children under 3 years but not in the older patients. This is the first report describing the molecular characterization of virulence genes in Campylobacter isolates from this region. The findings indicate that strains of different virulence genetic make-up are circulating in the population, with children under the age of 3 years being most vulnerable. Further work on the molecular characterization, gene expression and determination of the invasive phenotypes of $C$. jejuni strains circulating in different regions is needed.
\end{abstract}

\section{INTRODUCTION}

Campylobacter jejuni is now recognized as a leading bacterial cause of food-borne disease in both developed and developing countries (Butzler, 2004). The spectrum of disease may range from mild, self-limiting, non-inflammatory diarrhoea to severe, inflammatory, bloody diarrhoea with pyrexia, abdominal cramps, bacteraemia and faecal leukocytes (Sack et al., 2001). Extra-intestinal disease may also occur, particularly in those with underlying immunosuppression (Tee \& Mijch, 1998). A notable complication of C. jejuni infection is the development of Guillain-Barré syndrome, which has a significant association with serological evidence of recent previous infection with Campylobacter (Mishu et al., 1993). Several factors have been proposed to explain the varied clinical presentation associated with Campylobacter infection, and the phenotypic traits associated with different $C$.

tThese authors contributed equally to this work.

Abbreviations: BDFH, Bahrain Defence Force Hospital; Cdt, cytolethal distending toxin; GCC, Gulf Co-operation Council; iam, invasionassociated marker; SMC, Salmaniya Medical Complex. jejuni strains may be related to their genetic diversity. However, the precise mechanism of pathogenicity is yet to be elucidated fully.

Toxin production and the invasive capability of infecting strains may modulate the clinical presentation of Campylobacter enteritis. Cytolethal distending toxin (Cdt) production by $C$. jejuni was first described by Johnson \& Lior (1988). CdtB, which is encoded by the $c d t B$ gene, is the active subunit of the holotoxin, which causes cell distention and irreversible cell-cycle arrest (Eyigor et al., 1999). In addition to its toxigenic effect, it has also been suggested that Cdt may play a role in invasion (Konkel et al., 2001). The occurrence of inflammation, infiltration of the lamina propria by neutrophils and bacteraemia indicate that invasion is an important pathogenic mechanism in Campylobacter infection (Ketley, 1997). Another virulence gene linked with Campylobacter invasiveness is the invasion-associated marker (iam) gene. In vitro studies have shown that this chromosomal genetic marker of Campylobacter strains is associated preferentially with both adherence and invasion (Carvalho et al., 2001). 
The occurrence of Campylobacter as a cause of diarrhoeal illness in the Gulf Co-operation Council (GCC) countries has been shown to range from 1.6 to $28 \%$ (Sethi et al., 1989; al-Freihi et al., 1993; Akhter et al., 1994; Ismaeel et al., 2002). However, there are no data describing the genetic make-up of Campylobacter strains circulating in this population. Here, we report the molecular characterization of two virulence genes in C. jejuni strains isolated in the Kingdom of Bahrain and the relationship with clinical infection.

\section{METHODS}

Bacterial strains and growth conditions. From January 2002 to January 2004, 96 C. jejuni strains were isolated from the stools of in- and out-patients seen at the Bahrain Defence Force Hospital (BDFH) and Salmaniya Medical Complex (SMC), as well as outpatients attending primary healthcare facilities in the Kingdom of Bahrain. SMC and BDFH are national referral centres for specialist care, laboratory diagnosis and admissions. Symptomatic patients were defined as those who presented with diarrhoea (an increased number of loose bowel motions) with or without vomiting, fever and abdominal pain (Friedman \& Isselbacher, 1998). For each patient, the attending physician completed a questionnaire designed to collect socio-demographic data as well as information on clinical parameters such as type and duration of symptoms, including the presence of nausea, vomiting, abdominal pain and fever. Abdominal pain was classified as mild, diffuse and/or crampy according to clinical judgement. Diarrhoea was defined as watery, malabsorptive or bloody depending on the appearance of the stools. Previous administration of antibiotics or chemotherapy (within the preceding month) was recorded.

Stool samples were processed at the microbiology laboratories at BDFH and SMC according to guidelines set out in the Bahrain National Quality Control Manual. All samples were cultured for Salmonella, Shigella, Campylobacter, Aeromonas, Plesiomonas and Yersinia as described previously (Jamsheer et al., 2003). Campylobacter cultures were carried out using blood-free selective agar containing modified charcoal cefoperazone deoxycholate selective supplement (Oxoid). Plates were incubated at $42^{\circ} \mathrm{C}$ for $48 \mathrm{~h}$ under microaerobic conditions (CampyGen; Oxoid). Suspected colonies were subcultured on chocolate agar plates and incubated microaerobically at $37^{\circ} \mathrm{C}$ for 24 h (CampyGen; Oxoid). Negative cultures were reincubated for an additional 24 h. C. jejuni ATCC 33291 was used as a quality-control strain. Campylobacter isolates were identified to species level using routine biochemical tests (production of catalase, hippurate and indoxyl acetate), sensitivity to nalidixic acid and cephalotin and temperature preferences for growth at 37 and $42{ }^{\circ} \mathrm{C}$, as described previously (Ismaeel et al., 2002).

Microscopic assessment for parasites, cysts and ova was carried out by the formalin/ether concentration method and direct wet preparation (Ismaeel et al., 2002). All samples from children under the age of 3 years were tested for the presence of enteropathogenic Escherichia coli as well as rotavirus and adenovirus using the Diarlex Rota-Adeno card agglutination test kit (Orion Diagnostics). The viral investigations were carried out at the Public Health Laboratory, Bahrain. All bloody stools were examined for enterohaemorrhagic E. coli irrespective of the patient's age.

Detection of iam and cdtB genes by PCR. Template DNA for PCR was extracted using the Chelex 100 boiling method, as described previously (Ismaeel et al., 2005). Amplification of the iam locus was carried out in a mastermix volume of $40 \mu$ containing $1 \times$ DNA Taq polymerase buffer, $0.2 \mathrm{mM}$ each deoxynucleotide triphosphate,
2 U Taq DNA polymerase (Boehringer) and 30 pmol each of forward primer $1 \cdot 6 \mathrm{~F}$ (5'-GCGCAAAATATTATCACCC-3', nt 316-334 of the iam locus) and reverse primer 1.6R (5'-TTCACGACTACTATGCGG-3'; Interactiva $\mathrm{GmbH}$ ). The amplification program consisted of 30 cycles of $1 \mathrm{~min}$ at $94^{\circ} \mathrm{C}, 2 \mathrm{~min}$ at $42^{\circ} \mathrm{C}$ and $3 \mathrm{~min}$ at $72{ }^{\circ} \mathrm{C}$. The PCR product was detected by electrophoresis on a $2 \%$ agarose gel and visualized under UV light after staining with ethidium bromide. The $c d t B$ gene was detected as described previously (Ismaeel et al., 2005) using degenerate primers VAT2 and WMI1 [5'-GT(A/C/G/T)GC(A/C/G/T)AC(G/C/T)TGGAA(C/T)CT(A/G/ $\mathrm{C} / \mathrm{T}) \mathrm{CA}(\mathrm{A} / \mathrm{G}) \mathrm{GG}-3^{\prime}$ and $5^{\prime}-(\mathrm{G} / \mathrm{A}) \mathrm{TT}(\mathrm{G} / \mathrm{A}) \mathrm{AA}(\mathrm{G} / \mathrm{A}) \mathrm{TC}(\mathrm{A} / \mathrm{G} / \mathrm{C} / \mathrm{T})-$ CC(T/C)AA(T/G/A)ATCATCC-3', respectively; Interactiva].

Statistical analysis. Data were entered in Microsoft EXCEL and analysed using the SPSS version 12 statistical package. Statistical significance was calculated using a chi square test.

\section{RESULTS}

During the study period, $96 C$. jejuni strains were isolated. Fifty $(52 \%)$ strains were $c d t B^{+} / \mathrm{iam}^{+}, 30(31 \%)$ were $c d t B^{+} / \mathrm{iam}^{-}$and $16(17 \%)$ were negative for both genes. Fig. 1 shows the characteristic $495 \mathrm{bp}$ band for $c d t B$ and the $518 \mathrm{bp}$ band for iam following electrophoresis. Sixtynine per cent (66/96) of the patients were under the age of 3 years; $56 \%$ had faecal leukocytes and $12 \%$ had blood in the stool. None of the patients was found to have parasites in the stool and neither rotavirus nor adenovirus was detected. Apart from one patient with $c d t B^{+} /$iam $^{+}$C. jejuni who also had Shigella, and two patients with $c d t B^{+} /$iam $^{-}$strains with concomitant Salmonella infection, no other bacterial infection was detected.

The detection of strains positive for one or a combination of two virulence genes was significantly higher in children under the age of 3 years $(P<0.01$ and $P<0.001$, respectively; Table 1). Strains negative for both genes were not detected in the 3- to 15-year-old group and their detection in the other two age groups was comparable. Although there was a preponderance of male patients ( 57 males versus 39 females) and over $60 \%$ of strains with virulence genes were from males, the detection of strains negative for both genes was comparable in both sexes (Table 1). The majority of strains in all three virulence categories were isolated from patients of Bahraini nationality.

A total of 70 patients (73\%) was found to be symptomatic (Table 2). Symptomatic patients were defined as those presenting with diarrhoea (an increased number of loose bowel motions) with or without vomiting, fever and abdominal pain (Friedman \& Isselbacher, 1998). Asymptomatic patients were those without diarrhoea who either had nonspecific symptoms such as abdominal discomfort and flatulence or were being tested as part of a routine recruitment screening. No correlation was found with previous administration of antibiotics and none of the patients had received chemotherapy. No specific correlation was found between the type of diarrhoea (watery or bloody) and culture positivity for Campylobacter. Remarkably, all patients over the age of 3 years who were infected with strains positive for one or both virulence genes were symptomatic (Table 2). In 

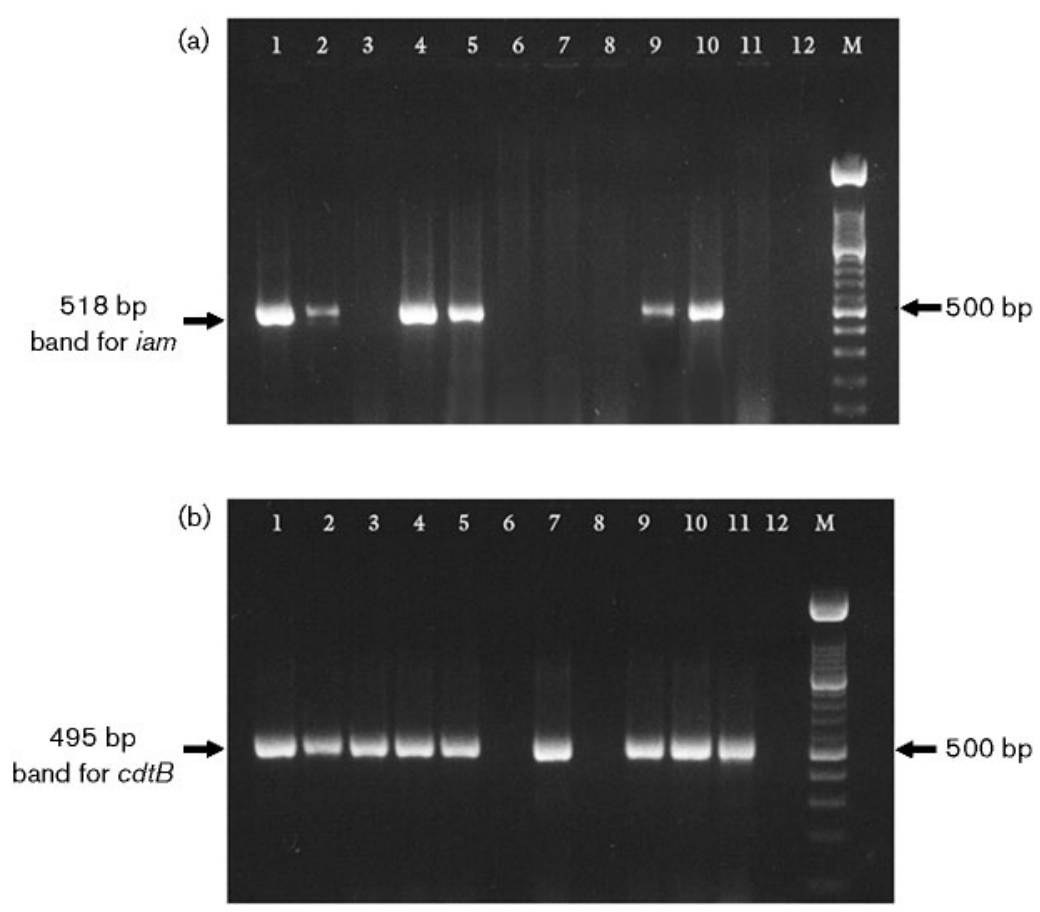

Fig. 1. (a) Electrophoresis of PCR products for the iam gene. Lanes 1 and 2 show the $518 \mathrm{bp}$ product from a known $\mathrm{iam}^{+}$control (in-house BDF 833 C. jejuni strain). Lanes $4,5,9$ and 10 show the results from strains positive for the iam gene. Lane M, $100 \mathrm{bp}$ marker. (b) Electrophoresis of PCR products for the $c d t B$ gene. Lanes 1 and 2 show the $495 \mathrm{bp}$ product from a known $\mathrm{cdt} \mathrm{B}^{+}$control (in-house BDF 14 C. jejuni strain). Lanes $3-5,7$ and $9-11$ show the results from strains positive for the $c d t B$ gene. Lane $\mathrm{M}, 100$ bp marker. contrast, a proportion of children under the age of 3 years infected with strains in these same virulence categories remained asymptomatic, although this was not statistically significant. Among the $16 \mathrm{cdtB} \mathrm{B}^{-} / \mathrm{iam}^{-}$strains identified, nine were isolated in children under the age of 3 years and the remaining seven strains were from those over the age of 15 years. In sharp contrast to the clinical picture observed for patients harbouring strains positive for one or two virulence genes, all those in the $>15$-year-old group who were positive for $c d t B^{-} /$iam $^{-}$strains were asymptomatic. However, these $c d t B^{-} /$iam $^{-}$strains resulted in significant clinically evident infections in the $<3$-year-old group $(P$ $<0.001$; Table 2) and the proportion of symptomatic infections was at a level comparable to those seen with $c d t B^{+} /$iam $^{+}$and $c d t B^{+} /$iam $^{-}$strains.

\section{DISCUSSION}

These findings provide data correlating the presence of virulence gene combinations in $C$. jejuni with the demographic and clinical parameters of the patients from whom the strains were isolated. In addition, this is the first report describing the molecular characterization of Campylobacter strains from the Arabian Gulf (GCC) region. A total of 96 patients with Campylobacter enteritis was identified during the study period and this low number probably reflects the fact that routine Campylobacter screening was only introduced in 2002 after a pilot study showed detection of this micro-organism in $1.6 \%(7 / 426)$ of children with diarrhoea (Ismaeel et al., 2002). It is possible that during the early phase of implementation of procedures for optimal

Table 1. Detection of $C$. jejuni strains with virulence genes among patients of different age groups, gender and nationalities

Percentages in parentheses indicate the proportion of $c d t B^{+} / i a m^{+}, c d t B^{+} / i a m^{-}$and $c d t B^{-} / i a m^{-}$strains found in each of the age group, gender and nationality categories. A significantly higher distribution of $c d t B^{+} / i a m^{+}$and $c d t B^{+} / i a m^{-} C$. jejuni strains was found among children under 3 years of age.

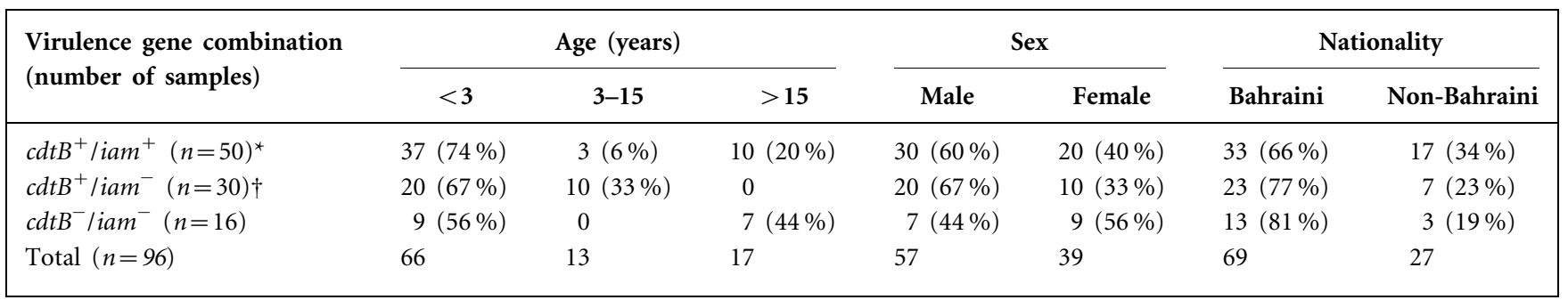

${ }^{\star} P<0 \cdot 001 ; \dagger P<0 \cdot 01$. 
Table 2. Distribution of symptomatic presentation among patients of different age groups with strains of different virulence categories

Symptomatic patients were defined as those who presented with diarrhoea with or without vomiting, fever and abdominal cramps. Numbers in parentheses indicate the number of symptomatic patients as a proportion of the total number of patients in that age group with $C$. jejuni strains of the corresponding virulence category. No significant difference in the number of symptomatic infections was seen in the different age groups with strains positive for one or both virulence genes. Compared with the other age groups, those in the $<3$-year-old age group with $c d t B^{-} /$iam $^{-}$strains had a significantly higher number of symptomatic infections.

\begin{tabular}{|c|c|c|c|}
\hline \multirow{2}{*}{$\begin{array}{l}\text { Strains with } \\
\text { virulence } \\
\text { genes }\end{array}$} & \multicolumn{3}{|c|}{$\begin{array}{l}\text { Symptomatic patients in } \\
\text { different age groups }\end{array}$} \\
\hline & $<3$ years & $3-15$ years & $>15$ years \\
\hline$c d t B^{+} /$iam $^{+}$ & $62 \%(23 / 37)$ & $100 \%(3 / 3)$ & $100 \%(10 / 10)$ \\
\hline$c d t B^{+} / \mathrm{iam}^{-}$ & $85 \%(17 / 20)$ & $100 \%(10 / 10)$ & $0 / 0$ \\
\hline$c d t B^{-} / \mathrm{iam}^{-}$ & $78 \%(7 / 9)^{\star}$ & $0 / 0$ & $0 \%(0 / 7)$ \\
\hline
\end{tabular}

${ }^{\star} P<0 \cdot 001$.

specimen collection, transport and analysis some cases might have been missed.

Strains with three combinations of virulence genetic makeup, $c d t B^{+} / \mathrm{iam}^{+}, c d t B^{+} / \mathrm{iam}^{-}$and $c d t B^{-} / \mathrm{iam}^{-}$, were identified, indicating that $C$. jejuni strains of diverse pathogenic potential are circulating in this population. The genes that we targeted are important for toxin production and invasiveness, both of which are pathogenicity mechanisms in Campylobacter infection (Ketley, 1997; Wassenaar, 1997). The $c d t B$ gene is associated with toxin production, and the clinical manifestations of Campylobacter enteritis are in keeping with the toxigenic effect of cytotoxins. Of particular clinical relevance is the fact that this toxigenic effect may be enhanced by pre-exposure to the antibiotics of choice for treatment of Campylobacter infection (Ismaeel et al., 2005). Furthermore, although studies have been carried out identifying these genes in individual Campylobacter strains, there is a paucity of data describing the molecular epidemiology of these virulence genes in clinical isolates obtained in different countries. Although in vitro work has shown that the iam gene is associated with adherence and invasion, there are only two reports describing the identification of this virulence gene in clinical isolates (Carvalho et al., 2001; Rozynek et al., 2005).

Diarrhoea is the hallmark of Campylobacter enteritis, but asymptomatic carriage of this pathogen has been described, particularly in developing countries where infection tends to be endemic (Blaser, 1997; Taylor et al., 1988; Ketley, 1997). Therefore, in this study only patients presenting with diarrhoea were classified as symptomatic. This is particularly relevant in children, where the occurrence of symptoms such as fever, vomiting and abdominal pain may be due to various viral illnesses and not indicative of Campylobacter enteritis. Indeed, the majority of patients were under the age of 15 years, in keeping with the fact that Campylobacter enteritis in non-industrialized nations is seen mainly in children, with attenuation of symptoms with increasing age (Blaser, 1997; Ketley, 1997). However, our findings showed that children under the age of 3 years are the most vulnerable population for Campylobacter infection, as 69\% (66/ 96) of the patients were in this age group and the strains isolated from these children were mostly positive for the virulence genes. This is consistent with reports that this is a high-risk age group for diarrhoeal illness of both viral and bacterial aetiology (Kosek et al., 2003). In addition, infection with strains negative for both virulence genes also resulted in symptomatic infection in this age group. This was in sharp contrast to the finding that older patients with $c d t B^{-} /$iam $^{-}$ strains were asymptomatic. Although the occurrence of symptoms with $c d t B^{-} /$iam $^{-}$strains might reflect the presence and expression of other virulence genes, we cannot completely rule out possible infection with other pathogens or false-negative results in the virological investigations. However, the fact that a wide spectrum of micro-organisms was investigated and that tests for rotavirus and adenovirus were carried out in a referral laboratory suggests that these scenarios are unlikely.

A proportion of children under the age of 3 years was found to be asymptomatic, even though strains positive for one or two virulence genes were isolated from their stools. Again, this is markedly different from the picture seen with the older age groups, where all of those infected with $c d t B^{+}$/ $\mathrm{iam}^{+}$and $\mathrm{cdtB} \mathrm{B}^{+} / \mathrm{iam}^{-}$strains were symptomatic. Breastfeeding, which protects against childhood enteric infections, is encouraged culturally in GCC countries and it is not unusual to find children being breastfed up to the age of 2 years (Alnasir, 1992; Ogbeide et al., 2004). In addition, a number of infant milk formulas on the market contain added prebiotics and probiotics, which are also protective against childhood diarrhoea (Senok et al., 2005). These factors could explain this reduced level of symptomatic infection in this very young age group.

The observed male preponderance in the study population was not statistically significant and therefore not suggestive of a gender-related susceptibility to infection. Furthermore, although there is a large expatriate population in the country, the majority of the patients were of Bahraini nationality and this probably reflects access to care rather than any real epidemiological difference.

This study provides baseline data on the molecular epidemiology of two C. jejuni virulence genes and the relationship with clinical Campylobacter infection. Although we have demonstrated the presence of these genes, their expression was not characterized. Differences in gene expression, which may occur as a result of mutations or interplay between host and environmental factors, may also explain the variation in symptomatic presentation in the age groups. However, 
although it has been suggested that C. jejuni may upregulate one set of genes whilst downregulating another during in vitro culture (Konkel et al., 1993), the precise mechanisms involved have not been clarified fully and it is yet to be determined whether this occurs in vivo. As the effect of any combination of genes may be strain dependent (with host and environmental factors influencing their expression), thus giving rise to differences in phenotypic appearance of virulence characteristics, further work on molecular characterization and gene expression, as well as the invasive phenotypes of $C$. jejuni strains circulating in different populations, is needed.

\section{ACKNOWLEDGEMENTS}

Partial support was provided by a grant from the Arabian Gulf University to G. A. B. as principal investigator. G. A. B. is supported by a Chair of Microbiology endowment of His Highness Shaikh Al-Jaber Al-Ahmad Al-Sabah.

\section{REFERENCES}

Akhter, J., Burdette, J. M., Qadri, S. M. \& Myint, S. H. (1994). Aetiology of gastroenteritis at a major referral centre in Saudi Arabia. J Int Med Res 22, 47-54.

al-Freihi, H., Twum-Danso, K., Sohaibani, M., Bella, H., el-Mouzan, M. \& Sama, K. (1993). The microbiology of acute diarrhoeal disease in the eastern province of Saudi Arabia. East Afr Med J 70, 267-269.

Alnasir, F. A. (1992). Knowledge and attitude of secondary schoolgirls towards breast-feeding in Bahrain. J Bahrain Med Soc 4, 6-10.

Blaser, M. J. (1997). Epidemiologic and clinical features of Campylobacter jejuni infections. J Infect Dis 176, S103-S105.

Butzler, J.-P. (2004). Campylobacter, from obscurity to celebrity. Clin Microbiol Infect 10, 868-876.

Carvalho, A. C. T., Ruiz-Palacios, G. M., Ramos-Cervantes, P., Cervantes, L.-E., Jiang, X. \& Pickering, L. K. (2001). Molecular characterization of invasive and noninvasive Campylobacter jejuni and Campylobacter coli isolates. J Clin Microbiol 39, 1353-1359.

Eyigor, A., Dawson, K. A., Langlois, B. E. \& Pickett, C. L. (1999). Cytolethal distending toxin genes in Campylobacter jejuni and Campylobacter coli isolates: detection and analysis by PCR. J Clin Microbiol 37, 1646-1650.

Friedman, L. S. \& Isselbacher, K. J. (1998). Diarrhea and constipation. In Harrison's Principles of Internal Medicine, pp. 236-244. Edited by A. S. Fauci, E. Braunwald, K. J. Isselbacher, J. D. Wilson, J. B. Martin, L. S. Kasper, S. L. Hauser \& D. I. Longo. New York: McGraw-Hill.

Ismaeel, A. Y., Jamsheer, A. E., Yousif, A. Q., Al-Otaibi, M. A. \& Botta, G. A. (2002). Causative pathogens of severe diarrhea in children. Saudi Med J 23, 1064-1069.
Ismaeel, A. Y., Senok, A. C., Bindayna, K. M., Bakhiet, M., Al Mahmeed, A., Yousif, A. Q. \& Botta, G. A. (2005). Effect of antibiotic sub inhibitory concentration on cytolethal distending toxin production by Campylobacter jejuni. J Infect 51, 144-149.

Jamsheer, A. E., Bindayna, K. M., Al-Balooshi, N. A. \& Botta, G. A. (2003). Trend of antibiotic resistance in 1316 Shigella strains isolated in Bahrain. Saudi Med J 24, 424-426.

Johnson, W. M. \& Lior, H. (1988). A new heat-labile cytolethal distending toxin (CLDT) produced by Campylobacter spp. Microb Pathog 4, 115-126.

Ketley, J. M. (1997). Pathogenesis of enteric infection by Campylobacter. Microbiology 143, 5-21.

Konkel, M. E., Mead, D. J. \& Cieplak, W., Jr (1993). Kinetic and antigenic characterization of altered protein synthesis by Campylobacter jejuni during cultivation with human epithelial cells. J Infect Dis 168, 948-954.

Konkel, M. E., Monteville, M. R., Rivera-Amill, V. \& Joens, L. A. (2001). The pathogenesis of Campylobacter jejuni-mediated enteritis. Curr Issues Intest Microbiol 2, 55-71.

Kosek, M., Bern, C. \& Guerrant, R. L. (2003). The global burden of diarrhoeal disease, as estimated from studies published between 1992 and 2000. Bull WH O 81, 197-204.

Mishu, B., Ilyas, A. A., Koski, C. L., Vriesendorp, F., Cook, S. D., Mithen, F. A. \& Blaser, M. J. (1993). Serologic evidence of previous Campylobacter jejuni infection in patients with the Guillain-Barre syndrome. Ann Intern Med 118, 947-953.

Ogbeide, D. O., Siddiqui, S., Al Khalifa, I. M. \& Karim, A. (2004). Breast feeding in a Saudi Arabian community. Profile of parents and influencing factors. Saudi Med J 25, 580-584.

Rozynek, E., Dzierzanowska-Fangrat, K., Jozwiak, P., Popowski, J., Korsak, D. \& Dzierzanowska, D. (2005). Prevalence of potential virulence markers in Polish Campylobacter jejuni and Campylobacter coli isolates obtained from hospitalized children and from chicken carcasses. J Med Microbiol 54, 615-619.

Sack, D. A., Lyke, C., McLauglin, C. \& Suwanvanichkij, V. (2001). Antibacterial Resistance in Shigellosis, Cholera and Campylobacteriosis. WHO/CDS/CSR/DRS/2001.8. Geneva: World Health Organization.

Senok, A. C., Ismaeel, A. Y. \& Botta, G. A. (2005). Probiotics: facts and myths. Clin Microbiol Infect 11, 958-966.

Sethi, S. K., Khuffash, F. A. \& al-Nakib, W. (1989). Microbial etiology of acute gastroenteritis in hospitalized children in Kuwait. Pediatr Infect Dis J 8, 593-597.

Taylor, D. N., Echeverria, P., Pitarangsi, C., Seriwatana, J., Bodhidatta, L. \& Blaser, M. J. (1988). Influence of strain characteristics and immunity on the epidemiology of Campylobacter infections in Thailand. J Clin Microbiol 26, 863-868.

Tee, W. \& Mijch, A. (1998). Campylobacter jejuni bacteremia in human immunodeficiency virus (HIV)-infected and non-HIV-infected patients: comparison of clinical features and review. Clin Infect Dis 26, 91-96.

Wassenaar, T. M. (1997). Toxin production by Campylobacter spp. Clin Microbiol Rev 10, 466-476. 